\title{
Synthesis of CdS Nanocrystals by Employing the By-Products of the Anaerobic Respiratory Process of Desulfovibrio alaskensis 6SR Bacteria
}

\author{
L. G. Rangel-Chávez, ${ }^{1}$ M. I. Neria-González, ${ }^{2}$ A. Márquez-Herrera, ${ }^{3}$ \\ M. Zapata-Torres, ${ }^{4}$ E. Campos-González, ${ }^{5}$ O. Zelaya-Angel, ${ }^{5}$ A. Guillen-Cervantes, ${ }^{5}$ \\ J. L. Fernandez-Muñoz, ${ }^{4}$ and M. Melendez-Lira ${ }^{5}$ \\ ${ }^{1}$ Departamento de Materiales, Universidad Autónoma Metropolitana Azcapotzalco, Avenida San Pablo 180, \\ Colonia Reynosa Tamaulipas, 02200 México, DF, Mexico \\ ${ }^{2}$ División de Ingeniería Química y Bioquímica, Tecnológico de Estudios Superiores de Ecatepec, Avenida Tecnológico S/N, \\ Colonia Valle de Anáhuac, 55210 Ecatepec de Morelos, MEX, Mexico \\ ${ }^{3}$ Departamento de Ingeniería Agrícola, DICIVA, Universidad de Guanajuato, Campus Irapuato-Salamanca, \\ Ex Hacienda el Copal km 9, Carretera Irapuato-Silao, 36500 Irapuato, GTO, Mexico \\ ${ }^{4}$ CICATA-IPN, Calzada Legaria No. 694, Colonia Irrigación, 11500 México, DF, Mexico \\ ${ }^{5}$ Departamento de Física, Centro de Investigación y de Estudios Avanzados del IPN, Apartado Postal 14-740, 07000 México, DF, Mexico
}

Correspondence should be addressed to M. I. Neria-González; ibineria@hotmail.com

Received 9 April 2015; Revised 2 June 2015; Accepted 9 June 2015

Academic Editor: Chuanbao Cao

Copyright (C) 2015 L. G. Rangel-Chávez et al. This is an open access article distributed under the Creative Commons Attribution License, which permits unrestricted use, distribution, and reproduction in any medium, provided the original work is properly cited.

\begin{abstract}
A novel methodology for the direct synthesis of CdS nanoparticles, using a biological agent that avoids the extracellular processing, and the results of the characterization of CdS nanocrystals are presented. The by-products of the anaerobic respiratory process of Desulfovibrio alaskensis 6SR along with aqueous solutions of Cd salts were successfully employed to produce CdS nanocrystals with mixed cubic and hexagonal phases. Nanocrystal size has a narrow size distribution with little dependence on the Cd concentration. Both the presence of the crystallographic cubic phase and the crystalline order decrease as Cd concentration increases. The band gap values obtained from optical transmission measurements are lower than those of the bulk crystal. Raman spectroscopy characterization agrees with electron transmission microscopy images and X-ray diffraction results indicating that the method promotes the formation of high structural quality nanocrystals when low concentrations of the Cd salt are used.
\end{abstract}

\section{Introduction}

Nanobiotechnology offers the use of microorganisms to synthesize a variety of inorganic nanoparticles with well-defined chemical composition, size, and morphology. This approach allows the development of ecofriendly technologies for the synthesis of materials with technological applications [1]. Cadmium sulfide (CdS) is a II-VI semiconductor with a band gap value of $2.42 \mathrm{eV}$ at room temperature. It has shown great potential for the manufacture of different devices such as second-generation photovoltaic panels, optical sensors, lightemitting diodes, and transistors [2-8]. Current approaches for synthesizing CdS as thin film or powder include different techniques, that is, hydrothermal/solvothermal routes, thermal decomposition, chemical bath deposition, laser ablation, and radio frequency sputtering. These may require multiple steps and energy-intensive process as thermal treatments at high temperature [9]. In general, to control both the size and dispersion size of the produced nanoparticles is cumbersome; additionally, conventional procedures are limited at small scale reactions. On the other hand, the use of cadmium for CdS production generates some resistance owing to the carcinogen effects associated with it. However, the wide range of applications of CdS nanoparticles has incentivized 
the search for new routes for its synthesis involving both low energy consuming processes and reduced production of toxic by-products.

Synthesis of nanoparticles using biological entities has gained great interest due to the capability of producing nanoand microlength scaled inorganic materials [10]. Moreover, biological methods are simpler and less expensive and allow a better control of the size and size dispersion of the nanoparticles [11]. The synthesis and assembly of nanoparticles using plants, bacteria, and fungi can be considered as "green chemistry" procedures. Unicellular and multicellular organisms are able to produce inorganic materials either intra- or extracellularly $[12,13]$. Sulfate reducing bacteria (SRB) are a group of microorganisms with anaerobic respiration; they are capable of using sulfates as electron acceptors and it is reduced to sulfide by oxidation of organic material. The production of hydrogen sulfide by SRB induces pitting corrosion on steel, turning SRB into a nuisance in some industries. However, the increase in the knowledge related to SRB has given place to several biotechnological applications. In the field of bioremediation, SRB are of great utility to remove heavy metals. The production of hydrogen sulfide by SRB allows the precipitation of metallic ions $\left(\mathrm{Me}^{2+}\right)$ present in contaminated effluents. This process is described by the following reactions:

$$
\begin{aligned}
& \underset{\left(\mathrm{e}^{-} \text {donor }\right)}{\operatorname{Organic} \text { matter }}+\underset{\left(\mathrm{e}^{-} \text {acceptor }\right)}{\mathrm{SO}_{4}{ }^{2-}} \\
& \longrightarrow 2 \mathrm{CH}_{3} \mathrm{COO}^{-}+\mathrm{HS}^{-}+\mathrm{HCO}_{3}^{-} \\
& \mathrm{Me}^{2+}+\mathrm{HS}^{-} \longrightarrow \mathrm{MeS} \downarrow
\end{aligned}
$$

The generation of sulfide diminishes the acidity promoting the precipitation of metals as insoluble metal sulfides that can be easily separated [14]. The species of the genus Desulfovibrio are the most studied in this field, showing a high efficiency in the removal of different metallic ions as $\mathrm{Zn}, \mathrm{Pb}$, $\mathrm{Cu}, \mathrm{Cd}, \mathrm{Ni}$, and $\mathrm{Cr}[15,16]$. There is evidence that nanoparticles prepared by biosynthesis are far superior in several ways to those prepared by chemical methods. In particular, for the biosynthesis of CdS nanoparticles, until now, most of the methods employed have had the drawback of requiring the presence of the cell, whereas CdS synthesis can be achieved inside or outside the cell $[17,18]$. The accumulation of $\mathrm{H}_{2} \mathrm{~S}$ in a biological reactor inhibits cellular proliferation; it has been estimated to show up at concentrations between 565 and $735 \mathrm{mgL}^{-1}$ [19-21]. The methodology proposed in this work would both improve the biological efficiency of the process and the production of higher purity CdS nanoparticles.

This paper highlights the use of the hydrogen sulfide generated by the anaerobic respiration of Desulfovibrio alaskensis 6SR to synthesize CdS nanoparticles with controllable size and crystallographic structure. Hydrogen sulfide production was achieved from a reactor where Desulfovibrio alaskensis 6SR was cultured, and then it was fed into a chemical reactor, containing a cadmium solution, to obtain $\mathrm{CdS}$. The procedure was carried out in anaerobic conditions under $\mathrm{N}_{2}$ atmosphere. The main goal of this work was to show that the CdS nanoparticles prepared from the hydrogen sulfide generated by the anaerobic respiration of Desulfovibrio alaskensis 6SR bacteria possess crystalline quality and physical properties either comparable to or better than those of the commercial material. The obtained material was characterized by X-ray diffraction, transmission electron microscopy, and optical transmission and Raman spectroscopies. The results showed that the process permits controlling the crystallographic structure through the Cd content in the solution. By employing low Cd salt contents, CdS samples with higher crystalline order are obtained and the growth of CdS crystals with cubic structure is promoted.

\section{Experimental Section}

Desulfovibrio alaskensis 6SR was growing on Postgate's medium C [22], which contained (g/L) $\mathrm{KH}_{2} \mathrm{PO}_{4}(0.5), \mathrm{NH}_{4} \mathrm{Cl}$ (1.0), $\mathrm{Na}_{2} \mathrm{SO}_{4}$ (4.5), $\mathrm{MgSO}_{4} \cdot 7 \mathrm{H}_{2} \mathrm{O}(0.06)$, sodium lactate (5.0), $\mathrm{CaCl}_{2} \cdot \mathrm{H}_{2} \mathrm{O}(0.06)$, yeast extract (1.0), sodium citrate (0.3), and $\mathrm{NaCl}(30.0)$. The medium was adjusted to $\mathrm{pH}$ 7.0 and $0.045 \mathrm{~L}$ of medium was placed into $0.12 \mathrm{~L}$ serum bottles. These vessels were capped with crimped aluminum butyl rubber stoppers and sterilized in an autoclave at $121^{\circ} \mathrm{C}$. Water $(1.20 \mathrm{~L})$ was heated to boiling point until reducing its volume to $1.0 \mathrm{~L}$; then the water was cooled at room temperature, under a stream of $\mathrm{N}_{2} ; 0.045 \mathrm{~L}$ oxygen-free water was dispensed into serum bottles and sealed. A cadmium acetate stock solution was prepared at $10 \mathrm{~g} / \mathrm{L}$ with respect to the ion $\mathrm{Cd}^{2+}$ and sterilized by membrane filtration (pore size: $0.22 \mu \mathrm{m}$ ); the water used to prepare this solution was oxygen-free water. Culture medium and cadmium solution were prepared and dispensed in anaerobic conditions under $\mathrm{N}_{2}$ (99.998\% purity) atmosphere. The bottles with oxygenfree water were spiked with the cadmium solution to obtain different concentrations of cadmium (170-400 mg/L or ppm). All used reagents were of analytical grade and were obtained from Sigma-Aldrich company.

A system of two reactors was employed to synthesize the CdS nanoparticles: a biological reactor for the culture and a chemical reactor, where the hydrogen sulfide and cadmium ions reacted to form the CdS; see Figure 1. CdS was obtained by centrifugation at $5000 \mathrm{rpm}$ for $15 \mathrm{~min}$; the powder was washed with a solution of acetone-water (4:1) following centrifugation at $5000 \mathrm{rpm}$ for $5 \mathrm{~min}$. The crystallographic properties of the samples were studied by X-ray diffraction (XRD) carried out in a Siemens D5000 system employing the $\mathrm{CuK} \alpha$ wavelength. The structure of the samples was observed by transmission electron microscopy (TEM), using a JEOL2010 system operated at $200 \mathrm{kV}$. Transmission measurements were done employing a lock-in standard technique using a $275 \mathrm{M}$ Acton single spectrometer equipped with 1200 lines/mm grating blazed at $500 \mathrm{~nm}$ and a silicon photomultiplier as detector. Powders were stuffed in a cylindrical hole with $2 \mathrm{~mm}$ height and $1 \mathrm{~mm}$ diameter. Micro-Raman measurements were carried out employing an HJY-LabRam system using the $632.8 \mathrm{~nm}$ line of a HeNe laser.

\section{Results and Discussion}

The XRD patterns of the samples obtained employing (a) $170 \mathrm{ppm}$, (b) $200 \mathrm{ppm}$, (c) $300 \mathrm{ppm}$, and (d) $400 \mathrm{ppm}$ of 


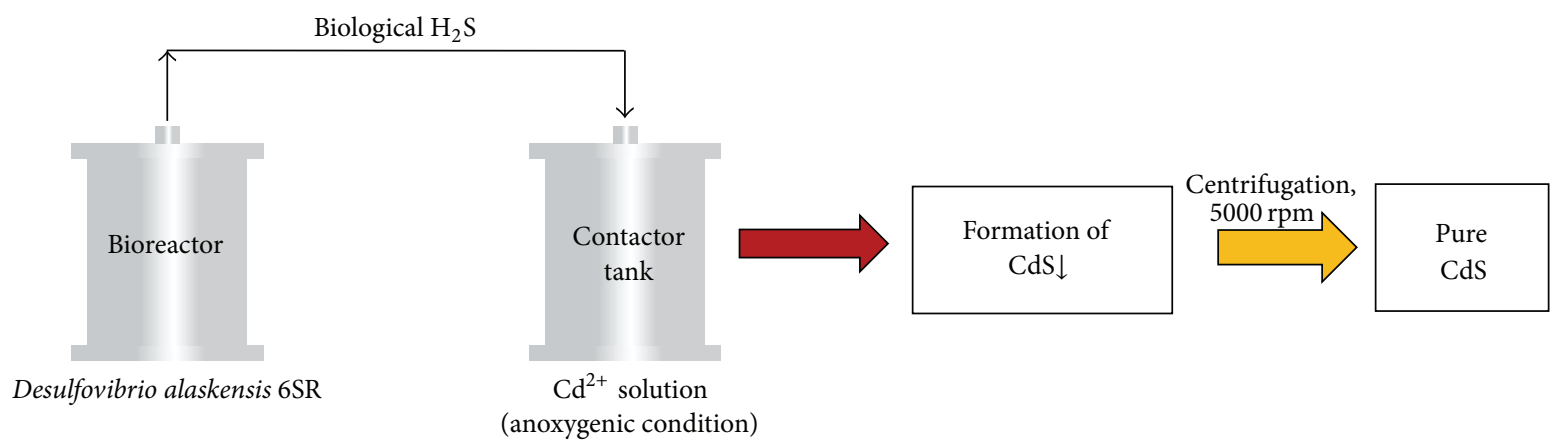

FIGURE 1: Experimental setup for the growth of the CdS nanoparticles.

TABLE 1: Crystal data for the cubic and hexagonal phase of CdS.

\begin{tabular}{|c|c|c|c|c|c|}
\hline Atom & Wyckoff position & $x$ & $y$ & $z$ & $\mathrm{ON}$ \\
\hline \multicolumn{6}{|c|}{ Cubic phase of CdS employed in the Powder Cell Program calculations: $a=5.811 \AA$, spatial group: F- $43 \mathrm{~m}(216), Z=4$. } \\
\hline $\mathrm{Cd}$ & $4 a$ & 0 & 0 & 0 & 1 \\
\hline S & $4 b$ & 0.25 & 0.25 & 0.25 & 1 \\
\hline \multicolumn{6}{|c|}{ Hexagonal phase of CdS employed in the Powder Cell Program calculations: $a=4.136 \AA, c=6.713 \AA$, spatial group: P63mc $(186) Z=2$. } \\
\hline $\mathrm{Cd}$ & $2 b$ & 0.3333 & 0.6667 & 0 & 1 \\
\hline S & $2 b$ & 0.3333 & 0.6667 & 0.37715 & 1 \\
\hline
\end{tabular}

ON: occupation number.

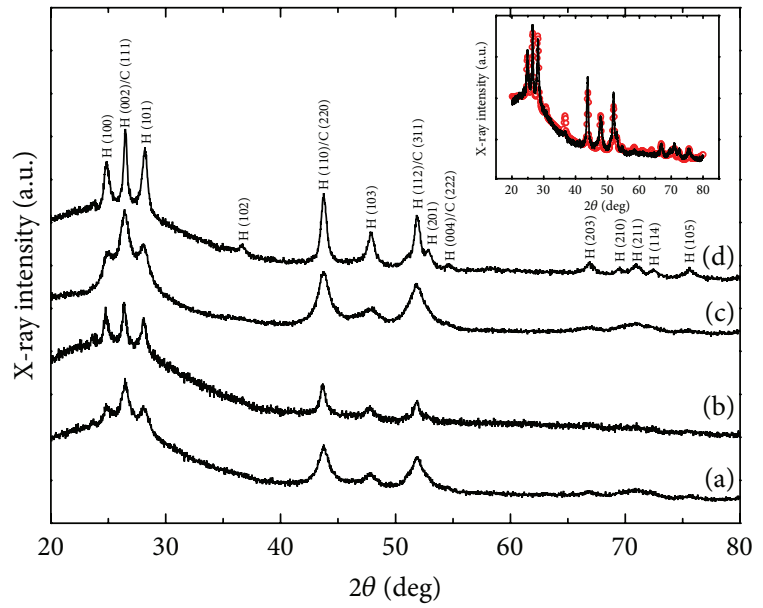

FIGURE 2: Diffractograms of CdS samples grown employing a solution with Cd concentration of (a) $170 \mathrm{ppm}$, (b) $200 \mathrm{ppm}$, (c) $300 \mathrm{ppm}$, and (d) $400 \mathrm{ppm}$. The inset shows representative fitting, represented by circles, employing the Rietveld methodology corresponding to sample $400 \mathrm{ppm}$.

the Cd salt solution are displayed in Figure 2. The diffraction peaks were indexed using the powder diffraction files JCPDF numbers 100454 and 4110419 for the cubic and hexagonal phases of CdS, respectively. No reflection was obtained related only to the CdS cubic phase for any sample. In all the X-ray patterns, the peaks related to the cubic phase overlapped with those related to the hexagonal phase. Both structural phases have the same density and composition; hence, the contribution of each phase to the peak intensity is related to its structure factors and multiplicities in the overlapping peaks. To determine the percentage of each phase, it was necessary to adopt a quantitative phase analysis by means of the Rietveld method. The used software was the Powder Cell software V. 2.4. The crystal data used in the quantitative phase analysis are given in Table 1 for the cubic and hexagonal phases of CdS, respectively $[23,24]$. The inset of Figure 2 shows representative X-ray diffractogram obtained by the Rietveld refinement for the sample grown with 400 ppm of the Cd salt; the continuous line corresponds to the experimental data, and circles symbols represent the calculated intensity obtained from the adjustment. To illustrate the quality of the fitting, the inset shows the region from 20 to 50 degrees. A similar adjustment was obtained for all samples. Table 2 shows the results obtained with the Powder Cell software for (a) the amount of each phase, (b) the grain size, and (c) the adjustment factor $R_{\mathrm{wp}}$. It can be seen that for all samples there is a hexagonal phase proportion of the order of 70 to 80 percent and a grain size around $15 \mathrm{~nm}$.

Figure 3 shows representative TEM micrographs for samples produced employing (a) $170 \mathrm{ppm}$ and (c) $400 \mathrm{ppm}$, together with the electron diffraction pattern, and high resolution transmission micrographs (HRTM) at selected areas for (b) $170 \mathrm{ppm}$ and (d) $400 \mathrm{ppm}$. For all studied samples, nanoparticles, whose shape was affected by the $\mathrm{Cd}$ content in the solution, were observed. While for the sample grown with $170 \mathrm{ppm}$ the shape of nanoparticles is irregular, it evolves toward an ellipsoidal shape for the sample grown with $400 \mathrm{ppm}$. The detailed analysis of the growth process of the crystals is far from the scope of this work but it is clear that shape evolution is influenced both by the amount of $\mathrm{Cd}$ ions available for the formation of CdS and the lack of spatial 

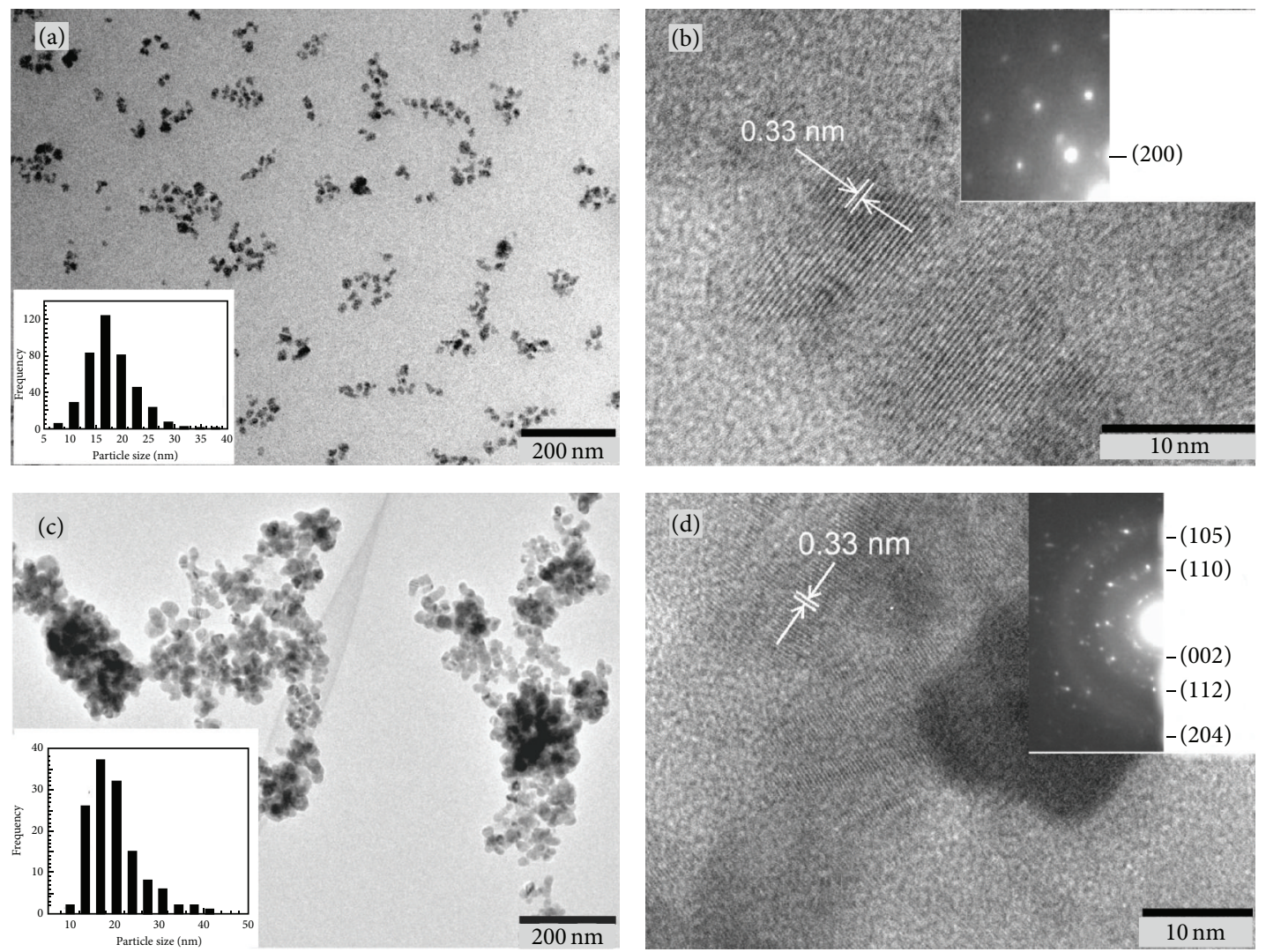

FIGURE 3: Left: transmission electron micrographics of samples: (a) $170 \mathrm{ppm}$ and (c) $400 \mathrm{ppm}$ along with the nanoparticles size distribution. Right: high resolution TEM micrographics for samples: (b) 170 ppm and (d) 400 ppm including the selected area electron diffraction pattern.

TABLE 2: Percent of phases, grain size, and adjustment factor $\left(R_{\mathrm{wp}}\right)$ as obtained from XRD analysis.

\begin{tabular}{lccccc}
\hline Sample & Cubic phase (\%) & Hexagonal phase (\%) & Grain size (nm) & $R_{\mathrm{wp}}(\%)$ & Band gap (eV) \\
\hline $170 \mathrm{ppm}$ & 30 & 70 & 17 & 10.3 & 6.2 \\
$200 \mathrm{ppm}$ & 17 & 83 & 17 & 11.1 & 2.226 \\
$300 \mathrm{ppm}$ & 27 & 73 & 10 & 7.5 & 2.220 \\
$400 \mathrm{ppm}$ & 22 & 78 & 15 & 2.146 \\
\hline
\end{tabular}

restrictions during crystal growth. High quality crystals were produced, as can be inferred from the crystalline planes observed by HRTM. In agreement with XRD results, TEM micrographs show a mixture of cubic and hexagonal phases for the whole set of samples studied. It is worth mentioning that the presence of cubic crystals with dimensions around 1$\mu \mathrm{m}$ was observed in TEM micrographs for the sample grown with $170 \mathrm{ppm}$, not shown here. Electron diffraction patterns indicate that those crystals grown using a solution with low $\mathrm{Cd}$ content depicted a greater order, suggesting that a tight control of the composition could promote the preferential growth of the cubic or the hexagonal phase. Electron diffraction patterns clearly show that crystalline disorder increases as the employed Cd concentration increases. These results are in agreement with reports of other authors indicating that the growth rate and sulfur content could influence the crystalline quality of crystals $[25,26]$. A deeper analysis of the micrographs was carried out in order to determine the size distribution and the crystal interplanar distances.
The particle sizes distribution (PSD) and the average size (Dp) of agglomerated nanoparticles were calculated using the ImageJ $1.46 c$ software. The number and weight average of the particle size $\left(D_{n}\right.$ and $D_{w}$, resp.) and polydispersity index in sizes (PDI) of the nanoparticles were calculated using a standard procedure [27].

The insets in Figures 3(a) and 3(c) show the PSD of $\mathrm{CdS}$ nanoparticles of samples grown employing 170 and $400 \mathrm{ppm}$, respectively. For sample grown using $170 \mathrm{ppm}$, it can be seen that a relatively narrow PSD can be obtained by this growth method with sizes ranging from 7 to $39 \mathrm{~nm}$. The corresponding $D_{n}$ was $18.7 \mathrm{~nm}$ with PDI of 1.1 (PDI describes the monodispersity of nanoparticles distribution). For the sample grown employing a concentration of $400 \mathrm{ppm}$ of the Cd salt, crystal sizes between 10 and $46 \mathrm{~nm}$ were obtained with $D_{n}$ and PDI values equal to $21.7 \mathrm{~nm}$ and 1.2, respectively. Because a value of PDI near to 1.0 implies a narrow and monodispersed distribution while higher values are associated with wider and polydispersed distributions, it 
is inferred that the PSDs obtained by this methodology are monodispersed. It is interesting to notice that the average size of the nanoparticles obtained from the study of TEM micrographs agrees closely with those reported in Table 2, obtained from XRD analysis.

Further structural characterization was carried out by high resolution TEM (HRTEM). Figures 3(b) and 3(d) showed the HRTEM image taken from a localized region within a single nanoparticle. The crystallographic planes observed showed that nanocrystals grown at $170 \mathrm{ppm}$ have a preferential growth direction while for the sample produced with higher $\mathrm{Cd}$ content different plane orientations are observed within the CdS nanocrystal. The insets in these figures show a selected area electron diffraction (SAED) pattern in which a ring structure typical of a polycrystalline structure can be observed $[28,29]$.

In agreement with the XRD results for the samples, the SAED patterns were indexed to Hawleyite cubic and Greenockite hexagonal CdS (JCPDF numbers 100454 and 411049). In Figure 3(b), the SAED pattern obtained from sample $170 \mathrm{ppm}$ is shown. The whole set of the allowed reflections for the hexagonal CdS crystal can be found in this ring pattern, indicating that the grains in the powders are randomly oriented. However, only the ring associated with the (200) cubic plane is labeled to emphasize the presence of the cubic crystalline phase. Figure 3(d) shows the SAED pattern of the $400 \mathrm{ppm}$ sample. Using ImageJ 1.46a software, the distance between lattice fringes was obtained. The $0.33 \mathrm{~nm}$ separation between the lattices fringes measured in both HRTEM images agrees well with the unit cell parameters of CdS. However, at this point, it is not possible to establish the crystallographic phase of the CdS nanocrystals because the interplanar separation of both the (111) planes of the cubic phase and the (002) planes of the hexagonal phase is the same.

The results reported until now indicated clearly that CdS nanocrystals were successfully prepared by employing the sulfide gas produced by the biological process of the sulfate reducing action of Desulfovibrio alaskensis 6SR. To further characterize the potential use of this material as a semiconductor in the diverse applications, it is necessary to characterize its electronic properties. Hence, to obtain the band gap value is fundamental to fully characterize the electronic properties of the material. Figure 4 presents the spectra for the studied samples after smoothing out the experimental spectra. The inset shows the spectrum for a commercial sample along with the curve obtained by fitting it, considering the simple model $T=I / I_{0}=e(-\alpha d)$ and considering the model of direct transitions between parabolic bands for the absorption coefficient:

$$
\begin{aligned}
& T=\frac{I}{I_{0}}=e(-\alpha d) \\
& \alpha=M\left(h \nu-E_{0}\right)^{1 / 2} .
\end{aligned}
$$

As usual, the symbols employed in (3) and (4) correspond to the transmission $(T)$, transmitted intensity $(I)$, incident intensity $\left(I_{0}\right)$, absorption coefficient $(\alpha)$, sample thickness $(d)$, transition matrix $(M)$, energy of the light beam $(h \nu)$, and

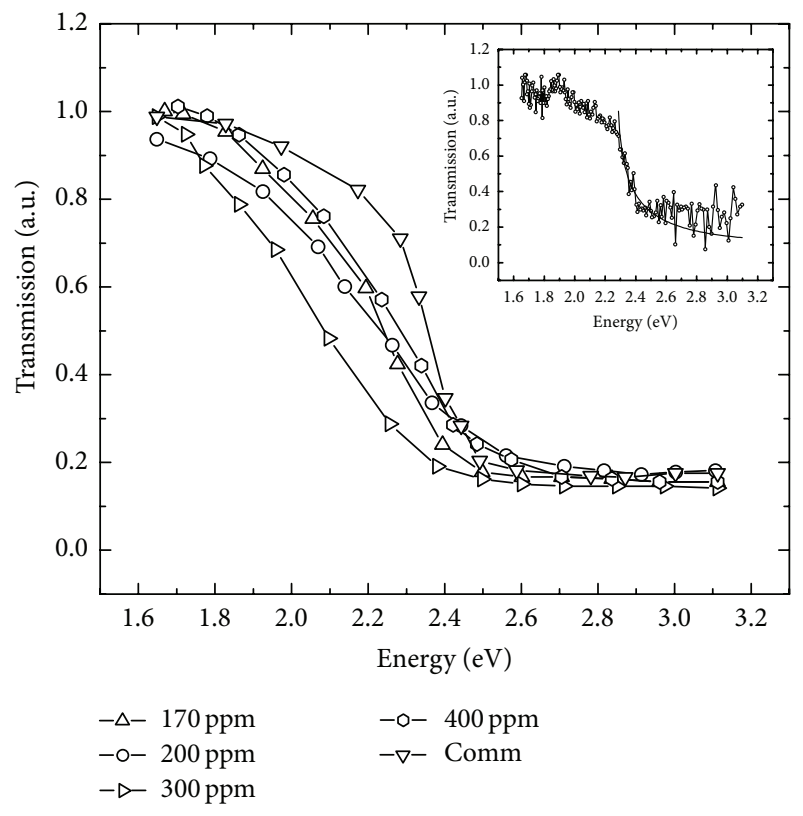

Figure 4: Optical transmittance of samples prepared with the Cd concentration indicated in the figure. Comm refers to the spectrum of a sample prepared with CdS powder from Sigma-Aldrich. The inset shows representative fitting to the model of direct transitions between parabolic bands along with the experimental spectrum.

band gap $\left(E_{0}\right)$. The fittings afford a reasonable approximation to the band gap value and are reported in Table 2. The band gap values are lower than the obtained ones for the commercial CdS powder and lower than the reported $2.42 \mathrm{eV}$ value of the bulk CdS crystal. It is worth noting that the band gap value of the nanocrystals decreases as the Cd salt content increases. Considering only the size of the CdS crystals determined by XRD analysis and clearly observed by TEM micrographs, the fact above mentioned is counterintuitive because the band gap should increase because of quantum confinement [30]. A possible explanation for the absence of quantum confinement effects could be that there is no capping material acting as confining barrier. Band gap values lower than those accepted for bulk CdS have been observed in thin films and are associated with the coexistence of the cubic and hexagonal phases [31]. In this case, besides the aforementioned possibility, the observed band gap reduction could be due to the fact that the surface of the CdS nanocrystals is not passivated; thus, the associated surface states will affect strongly the electronic properties of the material [32]. Marked reductions in the band gap value of $\mathrm{FeS}_{2}$ associated with surface states have been reported [33].

The structural quality of the materials plays an important role in devices performance; usually there is a compromise between the cost of production and the application. One of the advantages of CdS is that even the material produced with low crystalline quality is useful in the massive production of low cost photovoltaic devices. The approach shown here to produce CdS is a low cost alternative and, according to TEM results shown above, allows obtaining high quality material which could have a positive impact on the improvement of 


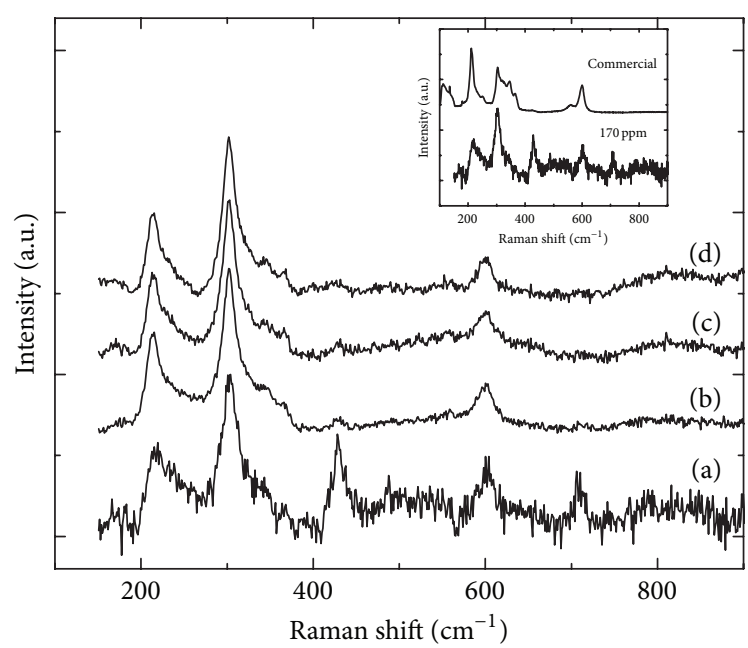

FIgURE 5: Normalized Raman spectra of the samples prepared with a solution with Cd concentration of (a) $170 \mathrm{ppm}$, (b) $200 \mathrm{ppm}$, (c) $300 \mathrm{ppm}$, and (d) $400 \mathrm{ppm}$. The inset shows the comparison of the Raman spectrum of the sample prepared with a solution with $\mathrm{Cd}$ concentration of $170 \mathrm{ppm}$ with that obtained using a commercial CdS sample.

the efficiency of the $\mathrm{CdTe} / \mathrm{CdS}$ photovoltaic heterostructure. Further structural information can be obtained by studying the vibrational properties through Raman spectroscopy. Phonons are associated with infinite crystals but it has been shown that Raman spectroscopy is also useful in the study of nanocrystals. However, theoretical developments are needed to produce models that take into account crystal imperfections $[34,35]$. The activation of phonons due to impurities, crystal size, and disorder effects in cadmium sulfide have been studied widely employing Raman spectroscopy [36-38].

Figure 5 shows the normalized Raman spectra of the samples studied here. The stronger features in all the spectra are located at 215 and $303 \mathrm{~cm}^{-1}$ related to the A1-TO and the $\mathrm{LO}$ mode, respectively, and $\mathrm{LO}$ overtone at $603 \mathrm{~cm}^{-1}$ [39]. Small peaks at 345 and $368 \mathrm{~cm}^{-1}$ become stronger for the samples grown with higher $\mathrm{Cd}$ salt content and could be associated with an increase in the structural disorder of the particles. The presence of bands associated with disorder around 100, 215, and $307 \mathrm{~cm}^{-1}$ has been clearly established and is also observed in the commercial CdS (see the inset in Figure 5). Raman spectrum of sample $170 \mathrm{ppm}$ presents additional features at 430 and $710 \mathrm{~cm}^{-1}$. The peak at $430 \mathrm{~cm}^{-1}$ is observed for the sample grown with the lowest salt content, but its strength decreased until it practically disappeared for the $400 \mathrm{ppm}$ sample. This peak could be an overtone of the $215 \mathrm{~cm}^{-1}$ peak, and its presence could be associated with the structural quality of this sample. It seems that the presence of cubic phase is more important in samples produced at low $\mathrm{Cd}$ salt concentrations. The additional features at 430 and $710 \mathrm{~cm}^{-1}$ could be phonons due to the CdS cubic phase; for now, this is highly speculative, and further work is under development.

\section{Conclusions}

CdS nanoparticles have been successfully prepared by employing the sulfide produced by anaerobic respiration of Desulfovibrio alaskensis 6SR. This approach keeps the advantages associated with the biosynthesis of materials, that is, low energy consumption, low amount of toxic residuals, and high structural quality, but overcomes the necessity of additional processing for separation of residues of intra- or extracellular tissue. The highest structural quality is obtained using lower concentrations of the $\mathrm{Cd}$ salt. The presented results suggest that CdS crystal quality may be controlled through the $\mathrm{Cd}$ salt concentration employed. The methodology adds value to the cadmium cleaning process by producing a semiconductor material with a potential use in the photovoltaic industry and it would be easily scaled up.

\section{Conflict of Interests}

The authors declare that there is no conflict of interests regarding the publication of this paper.

\section{Acknowledgments}

The partial financial support from CONACyT and SECITIDF is acknowledged. The authors thank the technical support of M. Guerrero, A. Garcia-Sotelo, L. Lopez, and D. PerezEscamilla. M. Zapata Torres thanks the hospitality of Dr. J. L. Peña Chapa during his sabbatical leave.

\section{References}

[1] S. R. Stürzenbaum, M. Höckner, A. Panneerselvam et al., "Biosynthesis of luminescent quantum dots in an earthworm," Nature Nanotechnology, vol. 8, no. 1, pp. 57-60, 2013.

[2] S. P. Mondal and S. K. Ray, "Cadmium sulfide nanostructures for photovoltaic devices," Proceedings of the National Academy of Sciences, India Section A: Physical Sciences, vol. 82, no. 1, pp. 21-29, 2012.

[3] K. W. Böer, "CdS enhances $\mathrm{V}_{\text {oc }}$ and fill factor in CdS/CdTe and CdS/CuInSe 2 solar cells," Journal of Applied Physics, vol. 107, no. 2, Article ID 023701, 2010.

[4] J. C. Ramos, I. Mejia, C. A. Martinez, and M. A. QuevedoLopez, "Direct on chip cadmium sulfide thin film transistors synthesized via modified chemical surface deposition," Journal of Materials Chemistry C, vol. 1, no. 40, pp. 6653-6660, 2013.

[5] D. Pathania, S. Sarita, and B. S. Rathore, "Synthesis, characterization and photocatalytic application of Bovine Serum Albumin capped cadmium sulphide nanopartilces," Chalcogenide Letters, vol. 8, no. 6, pp. 396-404, 2011.

[6] C. Li, J. Yuan, B. Han, and W. Shangguan, "Synthesis and photochemical performance of morphology-controlled CdS photocatalysts for hydrogen evolution under visible light," International Journal of Hydrogen Energy, vol. 36, no. 7, pp. 42714279, 2011.

[7] D. F. da Silva and D. Acosta-Avalos, "Light dependent resistance as a sensor in spectroscopy setups using pulsed light and compared with electret microphones," Sensors, vol. 6, no. 5, pp. 514-525, 2006. 
[8] S.-G. Hur, E.-T. Kim, J.-H. Lee, G.-H. Kim, and S.-G. Yoon, "Characterization of photoconductive CdS thin films prepared on glass substrates for photoconductive-sensor applications," Journal of Vacuum Science \& Technology B, vol. 26, no. 4, pp. 1334-1337, 2008.

[9] Y. Li, L. Tang, S. Peng, Z. Li, and G. Lu, "Phosphate-assisted hydrothermal synthesis of hexagonal CdS for efficient photocatalytic hydrogen evolution," CrystEngComm, vol. 14, no. 20, pp. 6974-6982, 2012.

[10] M. Wang, X. Liu, C. Cao, and C. Shi, "Synthesis of band-gap tunable $\mathrm{Cu}-\mathrm{In}-\mathrm{S}$ ternary nanocrystals in aqueous solution," $\mathrm{RSC}$ Advances, vol. 2, no. 7, pp. 2666-2670, 2012.

[11] J.-W. Moon, I. N. Ivanov, C. E. Duty et al., "Scalable economic extracellular synthesis of CdS nanostructured particles by a non-pathogenic thermophile," Journal of Industrial Microbiology \& Biotechnology, vol. 40, no. 11, pp. 1263-1271, 2013.

[12] P. Mohanpuria, N. K. Rana, and S. K. Yadav, "Biosynthesis of nanoparticles: technological concepts and future applications," Journal of Nanoparticle Research, vol. 10, no. 3, pp. 507-517, 2008.

[13] D. Mandal, M. E. Bolander, D. Mukhopadhyay, G. Sarkar, and P. Mukherjee, "The use of microorganisms for the formation of metal nanoparticles and their application," Applied Microbiology and Biotechnology, vol. 69, no. 5, pp. 485-492, 2006.

[14] T. Jong and D. L. Parry, "Removal of sulfate and heavy metals by sulfate reducing bacteria in short-term bench scale upflow anaerobic packed bed reactor runs," Water Research, vol. 37, no. 14, pp. 3379-3389, 2003.

[15] G. Cabrera, R. Pérez, J. M. Gómez, A. Ábalos, and D. Cantero, "Toxic effects of dissolved heavy metals on Desulfovibrio vulgaris and Desulfovibrio sp. strains," Journal of Hazardous Materials, vol. 135, no. 1-3, pp. 40-46, 2006.

[16] V. P. Utgikar, S. M. Harmon, N. Chaudhary, H. H. Tabak, R. Govind, and J. R. Haines, "Inhibition of sulfate-reducing bacteria by metal sulfide formation in bioremediation of acid mine drainage," Environmental Toxicology, vol. 17, no. 1, pp. 4048, 2002.

[17] X. Li, H. Xu, Z.-S. Chen, and G. Chen, "Biosynthesis of nanoparticles by microorganisms and their applications," Journal of Nanomaterials, vol. 2011, Article ID 270974, 16 pages, 2011.

[18] H. Bai, Z. Zhang, Y. Guo, and W. Jia, "Biological synthesis of size-controlled cadmium sulfide nanoparticles using immobilized rhodobacter sphaeroides," Nanoscale Research Letters, vol. 4, no. 7, pp. 717-723, 2009.

[19] M. I. Neria-González, J. C. Figueroa-Estrada, M. R. CruzDiaz, and R. Aguilar-López, "Adaptive smooth observer design for state estimation in Desulfovibrio alaskensis 6SR cultures," Revista Mexicana de Ingeniera Qumica, vol. 10, no. 1, pp. 137146, 2011.

[20] J. C. Figueroa, R. Aguilar, and M. I. Neria, "Analysis of unstructured kinetic modeling for a sulfate reducing process using Desulfobivrio alaskensis 6SR," BioTecnología, vol. 17, 2013.

[21] P. A. López-Pérez, M. I. Neria-González, L. B. Flores-Cotera, and R. Aguilar-López, "A mathematical model for cadmium removal using a sulfate reducing bacterium: Desulfovibrio alaskensis 6SR," International Journal of Environmental Research, vol. 7, article 501, 2013.

[22] J. R. Postgate, Sulfate-Reducing Bacteria, Cambridge University Press, New York, NY, USA, 1981.

[23] C.-Y. Yeh, Z. W. Lu, S. Froyen, and A. Zunger, "Zinc-blendewurtzite polytypism in semiconductors," Physical Review B, vol. 46, no. 16, Article ID 10086, 1992.
[24] A. Fakineos, A. W. Stevenson, and Z. Barnea, "The anharmonic temperature factor for crystals with the hexagonal wurtzite structure and its inclusion in models for least-squares refinement of intensity data," Australian Journal of Physics, vol. 35, no. 4, pp. 415-424, 1982.

[25] C. K. Kumar, N. T. Q. Hoa, S.-G. Yoon, E.-T. Kim, J.-H. Lee, and G.-H. Kim, "Highly photoconductive CdS thin films synthesized by using chemical bath deposition," Journal of the Korean Physical Society, vol. 55, no. 1, pp. 284-287, 2009.

[26] P. Rodríguez-Fragoso, J. Reyes-Esparza, A. León-Buitimea, and L. Rodríguez-Fragoso, "Synthesis, characterization and toxicological evaluation of maltodextrin capped cadmium sulfide nanoparticles in human cell lines and chicken embryos," Journal of Nanobiotechnology, vol. 10, article 47, 2012.

[27] A. Dumbrava, C. Badea, G. Prodan, and V. Ciupina, "Synthesis and characterization of cadmium sulfide obtained at room temperature," Chalcogenide Letters, vol. 7, no. 2, pp. 111-118, 2010.

[28] W. Jia and E. P. Douglas, "Characterization and size control of cadmium sulfide/cadmium disulfide nanoparticles within random ionomer solutions," Journal of Materials Chemistry, vol. 14, no. 4, pp. 744-751, 2004.

[29] L. Shi, C. Pei, and Q. Li, "Ordered arrays of shape tunable $\mathrm{CuInS}_{2}$ nanostructures, from nanotubes to nano test tubes and nanowires," Nanoscale, vol. 2, no. 10, pp. 2126-2130, 2010.

[30] S. Baskoutas and A. F. Terzis, "Size-dependent band gap of colloidal quantum dots," Journal of Applied Physics, vol. 99, no. 1, Article ID 013708, 2006.

[31] O. Zelaya-Angel, J. J. Alvarado-Gil, R. Lozada-Morales, H. Vargas, and A. Ferreira Da Silva, "Band-gap shift in CdS semiconductor by photoacoustic spectroscopy: evidence of a cubic to hexagonal lattice transition," Applied Physics Letters, vol. 64, no. 3, article 291, 1994.

[32] A. P. Alivisatos, "Perspectives on the physical chemistry of semiconductor nanocrystals," The Journal of Physical Chemistry, vol. 100, no. 31, pp. 13226-13239, 1996.

[33] F. W. Herbert, A. Krishnamoorthy, K. J. Van Vliet, B. Yildiz, and F. W. Herbert, "Quantification of electronic band gap and surface states on $\mathrm{FeS}_{2}$ (100)," Surface Science, vol. 618, p. 53, 2013.

[34] G. Gouadec and P. Colomban, "Raman spectroscopy of nanostructures and nanosized materials," Journal of Raman Spectroscopy, vol. 38, no. 6, pp. 598-603, 2007.

[35] M. P. Chamberlain, C. Trallero-Giner, and M. Cardona, "Theory of one-phonon Raman scattering in semiconductor microcrystallites," Physical Review B, vol. 51, no. 3, pp. 1680-1693, 1995.

[36] P. Y. Yu, M. H. Pilkuhn, and F. Evangelisti, "Resonant Raman study of Cl in CdS," Solid State Communications, vol. 25, no. 6, pp. 371-373, 1978.

[37] J. F. Scott and T. C. Damen, "Raman scattering from surface modes of small CdS crystallites," Optics Communications, vol. 5, no. 5, pp. 410-412, 1972.

[38] T. T. K. Chi, G. Gouadec, P. Colomban, G. Wang, L. Mazerolles, and N. Q. Liem, "Off-resonance Raman analysis of wurtzite CdS ground to the nanoscale: structural and size-related effects," Journal of Raman Spectroscopy, vol. 42, no. 5, pp. 1007-1015, 2011.

[39] X. L. Fu, L. H. Li, and W. H. Tang, "Preparation and characterization of CdS/Si coaxial nanowires," Solid State Communications, vol. 138, no. 3, pp. 139-142, 2006. 

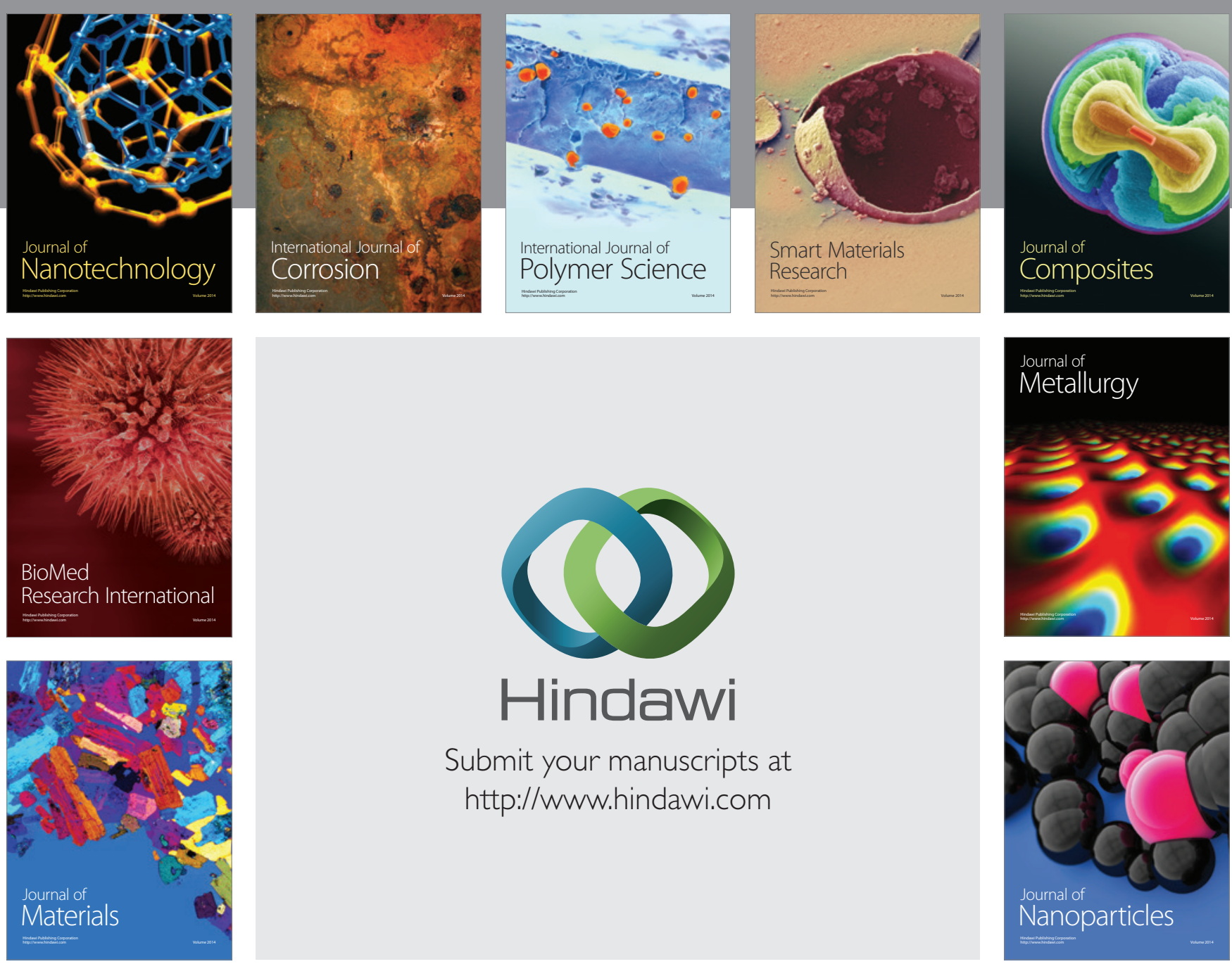

Submit your manuscripts at http://www.hindawi.com
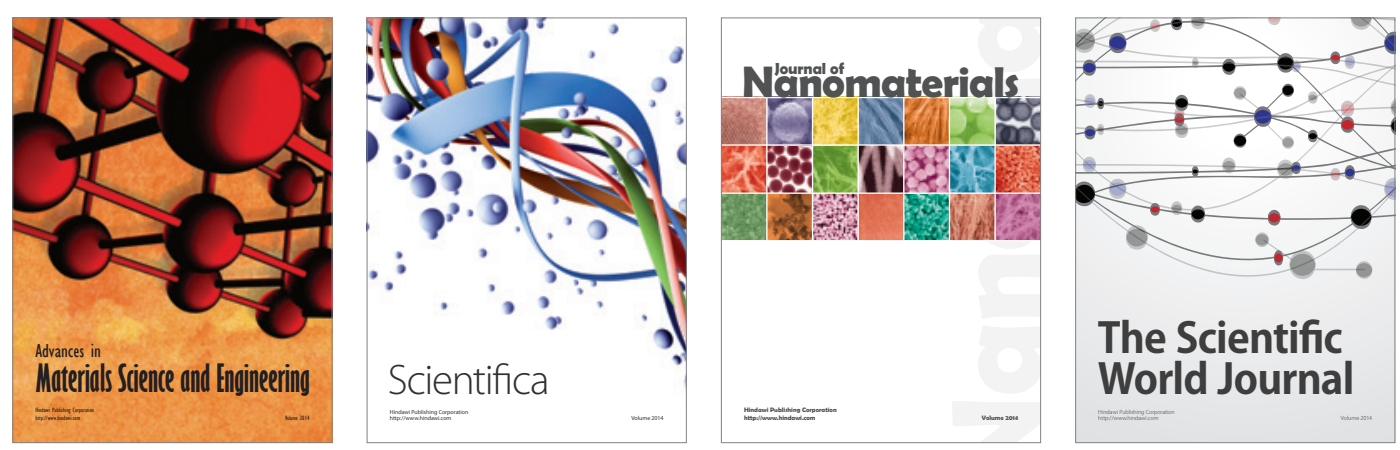

\section{The Scientific World Journal}
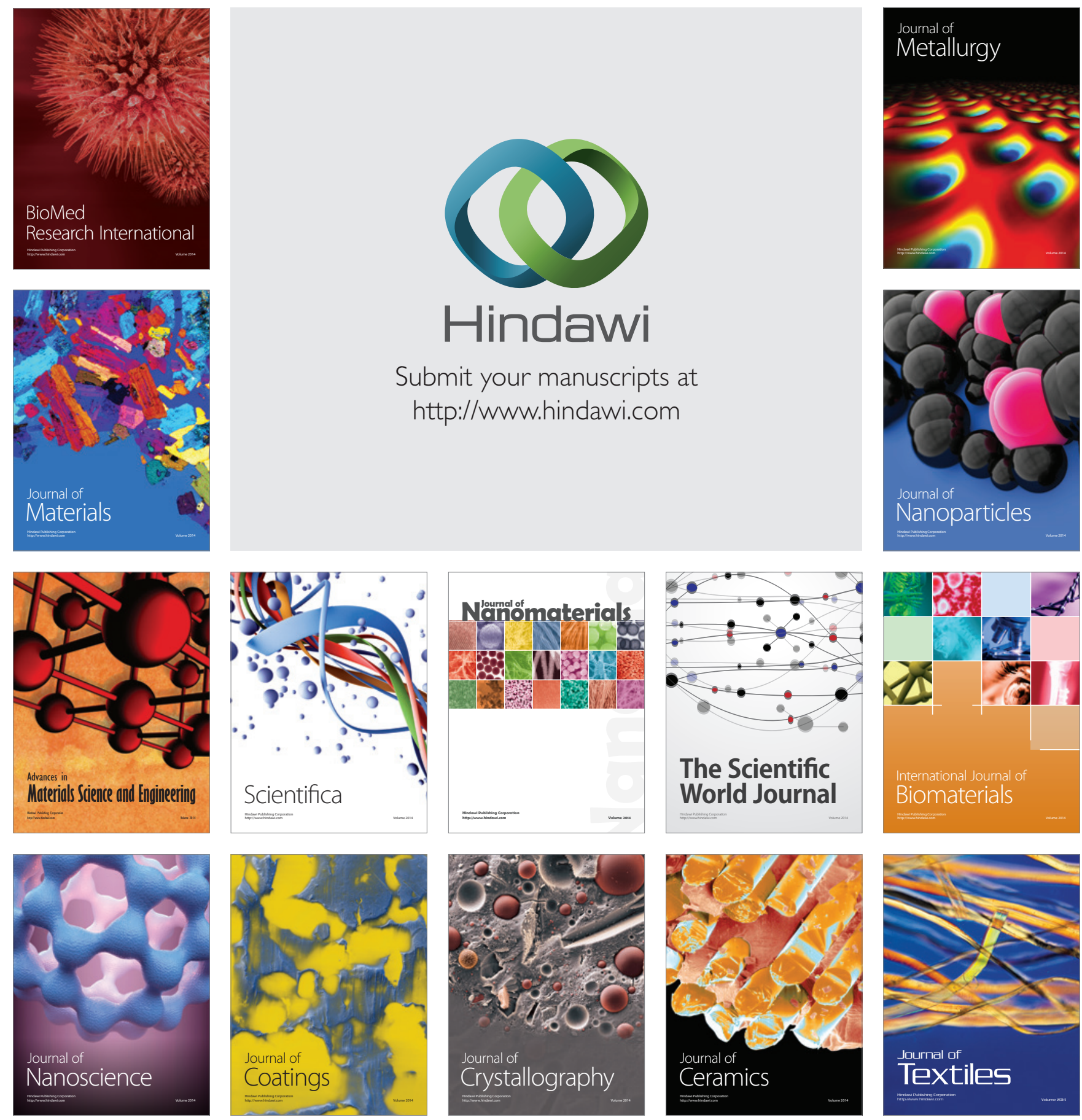